\title{
Challenges of Outward Facing Mobility Opportunities for Nursing Students: Pre and Peri Covid-19
}

\author{
Sheila CUNNINGHAM ${ }^{1}$
}

\section{Introduction}

The Covid-19 global pandemic has brought into stark relief our interdependence and relationship with other countries in many professional spheres including nursing and allied health professions. The global reach of nursing has grown both before this pandemic and will continue to grow exponentially. Irrespective of the makeup of societies nursing exists everywhere; whether it is based upon professional education and licensing or professional registration or employment systems. It is argued that nurses need to 'think globally' and that diversity; complexity; professional voice; leadership and inter disciplinary working are essential for global nursing $(1,2)$.

Regardless of location and societal or professional status, cultural diversity and sensitivity are essential nursing competencies $(3,4)$. However, while culturally appropriate care is essential and there is consensus on its need within the nursing literature, the evidence base for how best to develop cultural nursing competence is unclear (2). Comparing and contrasting our own health care systems such as with an international experience or mobility brings an appreciation of differences and possibilities in the wider, global context $(5,6)$. Arguably, COVID-19 has accelerated the need not only for an increased cultural awareness but an analysis of the differences; both positive and negative, when responding to global health issues.

Long term, attention to cultural competence in nursing programs is thought to provide students with deeper self-awareness with culturally diverse patients, colleagues and environments (5). Gosse and Katic - Duffy found "collaboration between ... partners promoted mutual cultural sensitivity, cultivated respect for diversity, enhanced awareness of health disparities and different health care systems". Collaboration also promotes critical thinking regarding complex health issues in resource- limited settings and supports the delivery of culturally competent care (6). 


\section{The benefits of international nursing placements}

The notoriously slow adoption of online learning in higher education in general has been galvanized by one dominant, single factor of COVID-19. An unprecedented acceleration to online learning and giving students continued access to their courses, emerged in a matter of days. This, one could argue, has been a catalyst to embedding new ways of working and learning longer term and opens the gates of innovation for vocational type programs. Radcliffe-Thomas et al (7) argue this is welcomed by students who appreciate studying in a 'borderless' online environment where they could hone their cultural awareness for their industry whilst building skills in digital literacy. This could be argued as one of the very few positives of the pandemic.

Although increasing online learning presents opportunities for global nurse education collaboration, the practical, immersive application of these skills is still best achieved through international clinical placements. The nursing literature is alive with personal accounts from students across the globe who have been transformed both professionally and personally through their international placement experiences. As with all placement experiences the potential for growth is astonishing (8). International placement contexts can add another dimension and accelerate this growth further (9). Core to this is the characteristic of experience in a 'high stakes' learning situation - central to international placements - where there is greater affective engagement by students. As their perspectives, resilience and adaptability are challenged so does the impact of the placement experience (8).

Students benefit tremendously from international exchange with overall increased chances of a higher degree classification and graduate level employment (10). Gains were particularly evident with black and minority ethnic (BAME) students, and those from a lower socio-economic background which reflect the nursing student population.

\section{The challenges of outward facing mobility opportunities}

The potential of outward facing opportunities is sporadic and yet to be fully realized especially in the United Kingdom (UK). These mobilities or exchanges under the Erasmus (or non-funded) route mean a period of exchange to a different host country either a university or clinical placement. In 2015-16 nursing had one of the lowest international mobility rates: $1.7 \%$ compared with a $7.2 \%$ average across the overall student population of the UK (10). Whilst this was pre-pandemic the current crisis has led to increased nursing student interest and recruitment and added pressures for broader and innovative placements. It is time to plan to capitalize and develop this. In the UK Go International's 'Stand Out' 
publicity campaign (10) aims to double the percentage of UK-domiciled students who study, work or volunteer abroad during their degrees. A key aim is to 'create a new generation of global graduates, and a higher education culture in which international opportunities are an aspiration for all students.

In the international context, despite compelling testimonies as to the transformative benefits of international placements, Browne and Fetherston (5) found profound variability from one education institution to the next. In addition, there was no clear consensus in the literature on what structures, support and assessments lead to greater student learning. Barriers to outward mobility are reported: insufficient funding, fear of isolation and security, language skills and the impact on friendships and relationships. However, it is the phenomena of culture shock that is most cited. Students report this as occurring mostly when they are unprepared for the different cultural norms and beliefs within their international placement. Therefore 'allowing students the time to debrief, and self-reflect is critical to process the experiences of learning from different cultures and health care system' (6). Practice learning specifically needs guidance and support to make learning explicit (8). This is a challenge in overtly different ways of working, practicing and support structures. In any country attitudes and values in mentors in the clinical learning environments has a powerful impact on student learning (11, 12) and an effective learning environment. The question remains then how one assures supportive quality environments especially of an international mobility placement experience.

\section{Quality indicators for international clinical placement learning: the HEALInt project}

Effective partnership working is core to successful transcultural teaching and embedding a global perspective in academic and practice learning. Clinical learning environments (CLE) abroad offer opportunities but are also subject to the same unpredictability as placements anywhere. Guidance emanating from UK professional bodies advocates assuring safety for students and staff, regular risk assessments, audits of the learning environment to confirm adequate levels of supervision and that planned experiences reflect the intended programme outcomes. While quality assurance of clinical learning environments is well established as a prospective approach in the UK (Nursing and Midwifery Council [NMC], 2008), documentation and processes for educational audit evaluation of CLE vary even between UK universities (13). There remains limited literature on proactive prospective measures in any other country or collective quality benchmarks. Saarikoski, Warne and others (14) challenged the quality of CLEs in Europe and developed the Clinical Learning Environment and Supervision Scale CLES and subsequent CLES $+\mathrm{T}$. These emphasised retrospective quality 
measurement via their widely validated evaluation tools (14). The challenge of developing a prospective audit and benchmark process in European CLEs lead partner universities from the UK, Spain, Malta, Finland and Poland to pursue an Erasmus+ funded project termed 'HEALINT'. This produced a robust panEuropean quality audit process for CLEs through establishing an International Quality Audit System for nursing and healthcare institutions hosting exchange nursing students. This was mapped to national and international priorities and other professional requirements. The intention is to ensure confidence in the quality of the clinical placement environments but also to promote and extend such opportunities and collaborative working. The dearth of existing or complex benchmarks in various countries offered an opportunity to positively contribute to this area. It drew together key documents and requirements, devised a shared joint benchmark and evaluation process. In addition, this collaborative process acts to review what a placement has to offer in respect of best practice in pedagogical support for nursing or healthcare students. It further facilitated opportunities to explore different cultural and practical nursing care environments.

This HEALint project is now an endorsed International Working Agreement (IWA) standards document. There are four overall project outputs available on the project website (www.healtint.eu).

1. Collate benchmark of quality indicators

2. Devise a clinical learning environment quality audit instrument

3. Outline training and guidance for audit processes

4. Evaluation of the tools and outputs

The project outputs are sustainable through the IWA endorsement but also through wider dissemination and use. This is now being expanded in an Erasmus + funded (HEAL4ALL) to other healthcare professionals undertaking international placement. In time it is hoped International placement experiences for healthcare professional students which build cultural awareness and competency is not an add-on option for the few but an opportunity for the many.

\section{Conclusion}

The global pandemic has meant many students placements in home countries were suspended not in the least, exchanges. Once placements resume as before this project and outputs provide a means to quality assure CLEs but seek to expand and strengthen international placement experiences. The importance of global nursing influences can be reflected in both the academic and practice component of courses via a macro-level perspective in the curriculum and a micro-level perspective on placement. Whilst the 'macro-level' can be experienced online (7) 
there remains the need for authentic, 'micro-level' clinical placements learning environments which can only be experienced in real life.

\section{References}

1. Thorne, S.E (2006) Nursing education: key issues for the twenty first century. Nurse Education Today 26: 614-621

2. Smith. N., Hennefer. D. L., Brettle. A., Jiang. X., Ho. R..k, Xiao. H. (2012) Nursing knowledge and skills for global employability: a systematic literature review and synthesis. University of Salford. [online] https:// usir.salford.ac.uk/id/eprint/22914/1/Global_Nurse_Employability_ PMI2_Connect.pdf

3. Papadopolous, I. (ed) (2006) Transcultural health and social care: development of culturally competent practitioners. London: Churchill Livingstone. Elsevier.

4. Leininger, M. (2002) Cultural care theory: a major contribution to advance transcultural nursing knowledge and practice. Journal of Transcultural Nursing 13(3): 189-192

5. Browne, C. A. and Fetherston, C. M. (2018) 'How do we facilitate international clinical placements for nursing students: A cross-sectional exploration of the structure, aims and objectives of placements', Nurse Education Today, 66, pp. 1-7.

6. Gosse, N. L. and Katic-Duffy, A. (2020) 'Nursing student and faculty perceptions of reciprocity during international clinical learning experiences: A qualitative descriptive study', Nurse Education Today, 84.

7. Radclyffe-Thomas, N., Peirson-Smith, A., Roncha, A., Lacouture, A. and Huang, A. (2018) 'Developing Global Citizenship: Co-creating Employability Attributes in an International Community of Practice', in Morley, D.A. (ed.) Enhancing Employability in Higher Education through Work Based Learning. Switzerland: Palgrave Macmillan, pp. 255-275.

8. Morley, D. A., Marchbank, P., Steyger, T., Taylor, L., Diaz, A. and Calleja, P. (2020) 'Designing and supporting extraordinary work experience', in Morley, D.A. and Golam, M.J. (eds.) Applied learning in higher education. Real world learning and innovation across the curriculum: Palgrave Macmillan.

9. Cunningham. S. (2014) A Global Learning Experience: Narratives of European Immersive Clinical Nursing Exchanges. The European 
Conference on Education (IAFOR) Official Conference Proceedings. [online] http://papers.iafor.org/wp-content/uploads/papers/ece2014/ ECE2014_03021.pdf Last accessed 20/09/20

10. Universities UK International (2018) Gone International: Expanding opportunities. Report on the 2015-16 graduating cohort. Available at:

11. https://www.universitiesuk.ac.uk/International/Documents/Gone $\% 20$ International_expanding\%20opportunities_digital.pdf

12. Salminen, L., Stolt, M., Saarikoski, M., Suikkala, A., Vaartio. H., LeinoKilpi. H., 2010. Future challenges for nurse education: a European perspective. Nurse Education Today. 30 (3), 233-238. https:// doi:10.1016/j.nedt.2009.11.004 .

13. Jokelainen, M. 2013. Mentorship in Placement Learning Environments. Systematic Review and Finnish and British Mentors Conceptions. Publications of the University of Eastern Finland Dissertations in Health Sciences 184, Kuopio. [online] Available: http://epublications.uef.fi/ pub/urn_isbn_978-952-61-1199-5/urn_isbn_978-952-61-1199-5.pdf Accessed 02/09/20.

14. Council of Deans for Health (2017) Outward student mobility for nurse, midwife and AHP students.

15. Hall, C., Higgins, C, Narayanasamy, M and Feliciano, S. (2019) Supporting Internationalisation of Traineeships in the Healthcare Sector (HEALINT) Briefing Paper. Development of the HEALINT Protocol. Available at: https://healint.eu/wp-content/uploads/sites/18/2020/03/ HEALINT-Briefing-Paper-Development-of-the-HEALINT-Protocol.pdf 